\title{
SLED CARRIERS AND MOLDED THREE-SURFACE TIPS FOR ATOMIC FORCE MICROSCOPE CANTILEVERS
}

\author{
R. P. Ried, H. J. Mamin, D. Rugar \\ IBM Research Division, Almaden Research Center \\ San Jose, CA 95120
}

\begin{abstract}
Novel sleds with silicon nitride atomic force microscope (AFM) cantilevers have been fabricated for an experimental AFM data storage system. The dimpled sleds slide over a surface and limit the maximum loading force on the AFM tip, thus allowing reduced constraints on the external loading system. Readback from a patterned rotating disk has been demonstrated using an AFM cantilever on a sled. Novel molded three-surface tips have been included on the cantilevers. The molded three-surface tips are expected to be more consistently sharp than conventional molded pyramidal tips.

\section{INTRODUCTION}

We have been investigating compact, high-density data storage using topographic readback by an AFM; conceptually the scheme may be likened to a tiny version of a phonograph with an AFM cantilever serving as a stylus [1]. Not surprisingly, maintaining a sharp AFM tip is a key issue. This paper addresses three aspects of the tip sharpness issue: improved loading force control, tip structure, and harder tip materials.
\end{abstract}

\section{SLEDS}

Air-bearing sliders are used to maintain gaps as low as $20 \mathrm{~nm}$ between a magnetic head and a spinning surface in magnetic disk drives. We wish to apply this air bearing technology to set the loading position of an AFM cantilever upon a surface, and thus improve upon the reliability and vibration immunity provided when a macroscopic actuator directly positions an AFM cantilever. Load control is particularly important for the AFM data storage application, where cantilever lengths of $10 \mu \mathrm{m}$ or less are desired to achieve high data rates [2]. Since in previous work we have observed negligible wear of a polycarbonate data disk under an AFM tip with low loading pressure, we have made silicon nitride sleds - which slide in contact with a data disk - as a first step toward making air-bearing sliders with AFM cantilevers.

Figure 1 is a scanning-electron microscope (SEM) image of the underside of a $370 \mu \mathrm{m}$-long, $140 \mu \mathrm{m}$-wide sled formed from $0.7 \mu \mathrm{m}$-thick silicon nitride. The sled is connected to a glass support chip by two compliant, $390 \mu \mathrm{m}$-long silicon nitride tethers. The perimeter of the sled is covered with smooth corrugations that serve three functions: 1) stiffen the sled, 2) provide a smooth surface for the sled to ride over peaks of a patterned medium, 3) plow contaminating debris from the path of the oncoming AFM tip. An " $\mathrm{H}$ " shaped AFM cantilever is in the center of the sled. The AFM tip is on the center bar of the "H". Ideally, the AFM tip should project slightly further out of plane than the corrugations so that when the sled is flat on a patterned medium, the AFM tip contacts the surface with a small applied loading force. Interdigitated fingers are located at the far end of the cantilever for interferometrically measuring cantilever deflection [3]. The sleds are fabricated in a similar way to the original silicon nitride AFM cantilevers [4].

In operation, the glass support chip is canted roughly 6 degrees with respect a spinning patterned medium. The support

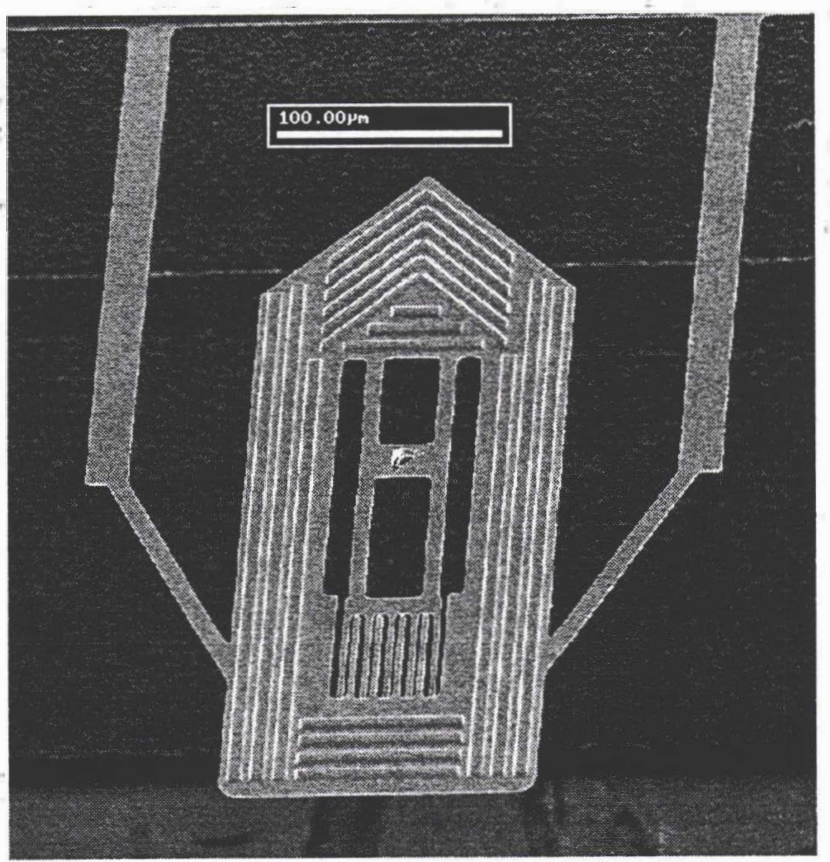

Figure 1. SEM image of corrugated sled with integral AFM cantilever and interdigitated fingers for interferometric readout. The sled is attached to a glass support chip via the tethers. The AFM tip projects beyond the corrugation depth in order to make contact when the sled is pressed flat on a data medium.

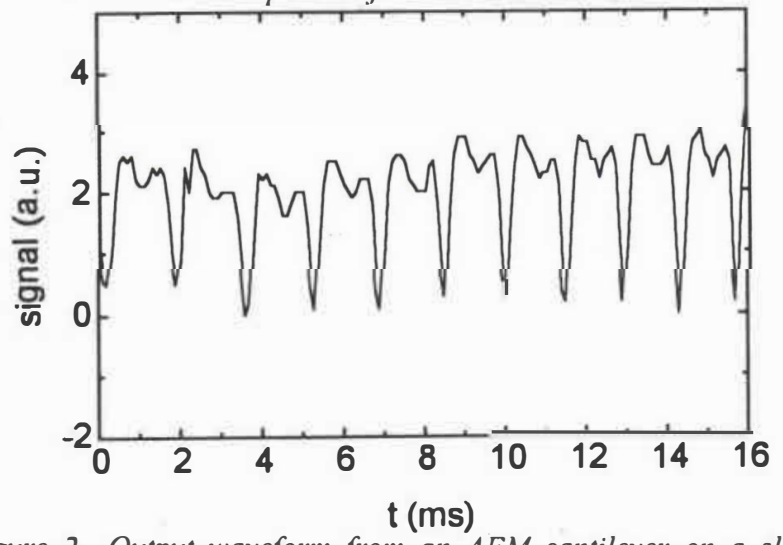

Figure 2. Output waveform from an $A F M$ cantilever on a sled riding over $60 \mathrm{~nm}$-deep, $0.5 \mu \mathrm{m}$-wide grooves with 5 to $9 \mu \mathrm{m}$ spacing on a spinning polycarbonate disk.

chip is initially brought toward the medium, so that the far end of the sled contacts the medium, and then moved closer so that the tethers bend and the sled rides flat on the surface. Further reduction in the spacing between the support chip and medium results in an increased loading force on the corrugations, with no increase in the AFM tip loading force expected.

Figure 2 shows a waveform obtained by optical beamdeflection measurements from an AFM cantilever on a sled riding over $60 \mathrm{~nm}$-deep, $0.5 \mu \mathrm{m}$-wide grooves with 5 to $9 \mu \mathrm{m}$-spacing on a spinning polycarbonate disk. Thus, proof of concept has been suc- 
cessfully demonstrated. The varying base line in Fig. 2 is attributed to disk height variations and/or a spurious bending mode of the sled. Further testing to characterize the response is in progress.

\section{MOLDED THREE-SURFACE TIPS}

The four silicon $\{111\}$ planes which are used to define a conventional molded AFM tip are prone to converge to a wedge rather than to a single point. The wedge shaped tip is duller than a perfect pyramidal tip. We have developed novel molded three-surface tips which converge toward a single point and which have a higher aspect ratio than the conventional pyramidal tip. Sharp, singlecrystal-silicon tips defined by three planes were developed in previous work [2].

Figure 3 outlines the process flow for forming a molded three-surface tip. In Fig. 3a, a high aspect-ratio trench with a curving mask shape is etched into a silicon (001) wafer, and then the trench is filled with silicon dioxide. Next, in Fig. 3b, the surface oxide is patterned as shown. In Fig. 3c, a wet anisotropic silicon etch is performed to leave two $\{111\}$ planes meeting at the vertical oxide sidewall of the trench to form a tip mold. Subsequently, the mold is filled with a material such as silicon nitride, cantilevers are patterned, and the silicon wafer and oxide trench are etched away.

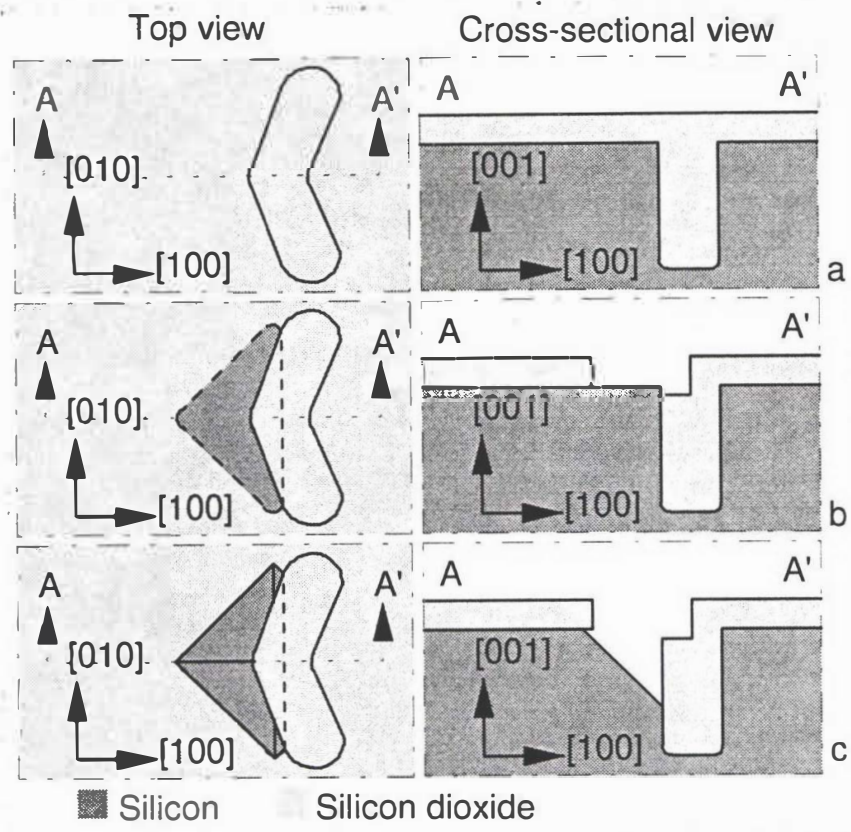

Figure 3. Fabrication of mold for three-surface tip. a) High aspect-ratio trench formation and filling trench with oxide. b) Pattern surface oxide. c) Wet anisotropic etch of silicon.

Figure 4 is an SEM image of the molded three-surface tip on the sled shown in Fig. 1. The measured tip radius of curvature is roughly $20 \mathrm{~nm}$. We believe that that the tip was dulled by the finite etch rate of the silicon nitride in the long hydrofluoric acid etch used to remove the $3 \mu \mathrm{m}$-thick oxide trench. Tip sharpness could be improved with the use of a thinner oxide trench or a different tip material. Other possible tip variations include oxide sharpening of the mold [5], and modifying the masking patterns so as to produce a pyramidal tip with a cylindrical hole in the center.

\section{DIAMOND TIPS}

Arrays of diamond tips have been successfully fabricated by

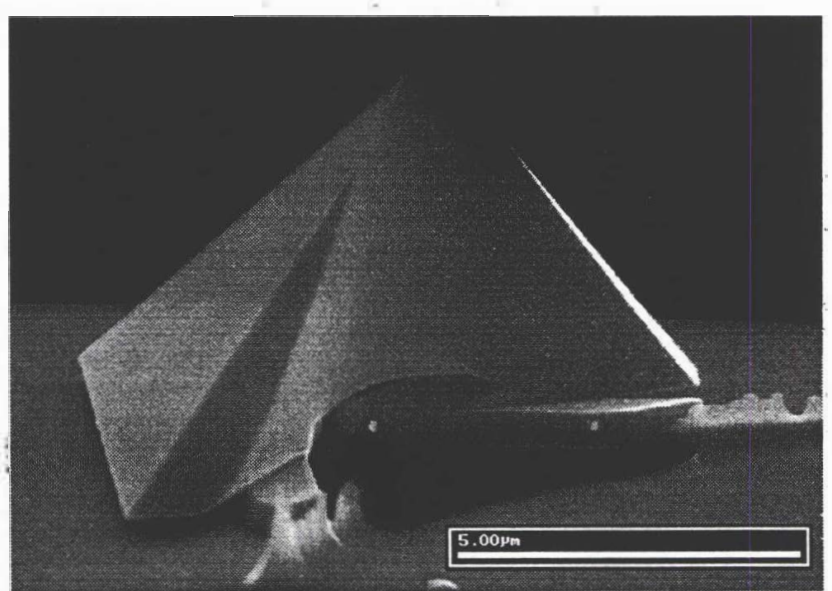

Figure 4. SEM image of molded three-surface tip from sled of Fig. 1. The vertical curving surface and one of the $\{111\}$ planes which define the tip are visible. Two ancillary $\{111\}$ planes are also visible on the structure. The shorter, high aspect-ratio ridges in the foreground arise from incomplete oxide filling of the trench.

depositing a $1 \mu \mathrm{m}$-thick layer of polycrystalline diamond [6] into pyramidal silicon molds and subsequently removing the silicon with tetramethyl ammonium hydroxide (TMAH). SEM measurements have shown that the radius of curvature of the tips are as low as $20 \mathrm{~nm}$.

Future work may include integration of pyramidal and threesurface diamond tips onto cantilevers, sleds, and air-bearing sliders to investigate the increased durability of diamond tips for AFM data storage applications.

\section{ACKNOWLEDGEMENTS}

This work is supported in part by DARPA contract number DABT63-95-C-0019. Devices were partially fabricated at the Stanford Nanofabrication Facility.

\section{References}

1. H. J. Mamin, B. D. Terris, L. S. Fan, S. Hoen, R. C. Barrett, and D. Rugar, "High-density data storage using proximal probe techniques,”' IBM J. Res. Dev., vol. 39, pp. 681-699, 1995.

2. R. P. Ried, H. J. Mamin, B. D. Terris, L. S. Fan, and D. Rugar, "6 MHz, $2 \mathrm{~N} / \mathrm{m}$ piezoresistive cantilevers with INCISIVE tips,"' IEEE J. Microelectromech. Syst., vol. 4, pp. 294-302, 1997.

3. S. R. Manalis, S. C. Minne, A. Atalar, and C. F. Quate, "Interdigital cantilevers for atomic force microscopy," Appl. Phys. Lett., vol. 69, pp. 3944-3946, 1996.

4. T. R. Albrecht, S. Akamine, T. E. Carver, and C. F. Quate, "Microfabrication of cantilever styli for the atomic force microscope,' J. Vac. Sci. Technol. A, vol. 8, pp. 3386-3396, 1990.

5. S. Akamine and C. F. Quate, "Low temperature thermal oxidation sharpening of microcast tips," J. Vac. Sci. Technol. $B$, vol. 10, pp. 2307-2310, 1992.

6. Films deposited at Crystallume Inc., Santa Clara, CA. 